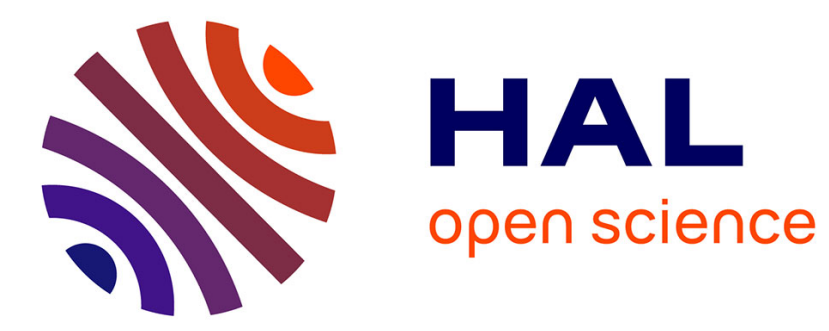

\title{
Pompage direct des paires d'ions Cr3+ dans le rubis
}

\author{
M. Ben Ghorbal, E. Duval, Bernard Champagnon
}

\section{To cite this version:}

M. Ben Ghorbal, E. Duval, Bernard Champagnon. Pompage direct des paires d'ions Cr3+ dans le rubis. Journal de Physique Lettres, 1975, 36 (4), pp.101-103. 10.1051/jphyslet:01975003604010100 . jpa-00231158

\section{HAL Id: jpa-00231158 https://hal.science/jpa-00231158}

Submitted on 1 Jan 1975

HAL is a multi-disciplinary open access archive for the deposit and dissemination of scientific research documents, whether they are published or not. The documents may come from teaching and research institutions in France or abroad, or from public or private research centers.
L'archive ouverte pluridisciplinaire HAL, est destinée au dépôt et à la diffusion de documents scientifiques de niveau recherche, publiés ou non, émanant des établissements d'enseignement et de recherche français ou étrangers, des laboratoires publics ou privés. 


\title{
POMPAGE DIRECT DES PAIRES D'IONS $\mathrm{Cr}^{3+}$ DANS LE RUBIS
}

\author{
M. BEN GHORBAL, E. DUVAL et B. CHAMPAGNON
}

Laboratoire de Spectroscopie et de Luminescence, Université Claude-Bernard, Lyon, I 69621 Villeurbanne, France

(Reçu le 12 décembre 1974, révisé le 21 janvier 1975, accepté le 5 février 1975)

\begin{abstract}
Résumé. - L'excitation des raies $R, N_{1}, N_{2}$ a été mesurée dans la région de la bande $U$, à basse température. Les raies à zéro-phonon des transitions ${ }^{4} \mathrm{~A}_{2}{ }^{4} \mathrm{~A}_{2} \rightarrow{ }^{4} \mathrm{~A}_{2}{ }^{4} \mathrm{~T}_{2}$ ont été mises en évidence à $6055 \AA$ pour les quatrièmes voisins appariés et à $6084 \AA$ pour les troisièmes voisins appariés. Ces mesures confirment le pompage intrinsèque des paires.
\end{abstract}

\begin{abstract}
The excitation of $R, N_{1}, N_{2}$ lines has been measured in the region of the $U$ band, at low temperature. The zero-phonon lines of ${ }^{4} \mathrm{~A}_{2}{ }^{4} \mathrm{~A}_{2} \rightarrow{ }^{4} \mathrm{~A}_{2}{ }^{4} \mathrm{~T}_{2}$ transitions have been found at $6055 \AA$ for the 4th-neighbour pairs and at $6084 \AA$ for the 3rd-neighbour pairs. These measurements confirm the intrinsic pumping in the pairs.
\end{abstract}

Il est bien connu [1, 2] que lorsqu'on augmente la concentration en ions $\mathrm{Cr}^{3+}$ dans le rubis, de nouvelles raies d'émission s'ajoutent aux raies $R_{1}(6936 \AA)$ et $\mathbf{R}_{2}(6922 \AA)$ du rubis peu dopé (substitution de moins de $0,1 \%$ d'ions $\mathrm{Cr}^{3+}$ dans $\alpha-\mathrm{Al}_{2} \mathrm{O}_{3}$ ). Ces raies n'ont pas été prévues par la théorie du champ cristallin [3]. Elles sont attribuées aux ions $\mathrm{Cr}^{3+}$ liés par interaction d'échange pour former des paires [4]. Les deux raies les plus intenses sont généralement repérées par $\mathrm{N}_{1}(7041 \AA)$ due aux ions $\mathrm{Cr}^{3+}$ interagissant avec le troisième plus proche voisin et par $\mathrm{N}_{2}(7009 \AA)$ due aux ions $\mathrm{Cr}^{3+}$ liés au quatrième plus proche voisin [5-7].

Les études des propriétés optiques des cristaux de rubis fortement dopé et des effet 、 que produit l'interaction d'échange sur les niveau des ions $\mathrm{Cr}^{3+}$ liés par paire, ont été développées "ssentiellement pour le niveau fondamental ${ }^{4} \mathrm{~A}_{2}$ et pour le premier niveau excité ${ }^{2} \mathrm{E}$ [8-9]. Très peu d'attention a été ıçcordée à l'étude des autres niveaux excités que présente le rubis fortement dopé, cela étant dû au fait, que l'on supposait que l'interaction d'échange $i \epsilon$ fait que déplacer très peu ces niveaux et n'intrcauit aucun changement radical sur eux, et à "'ypothèse d'Imbush [10] qui prétend que le pompage sur les niveaux d'énergie des paires ne se fait que par transfert d'énergie à partir des niveaux des ions $\mathrm{Cr}^{3+}$ isolés.

Récemment T. Kushida et Y. Tanaka [11] utilisant un laser à fréquence variable ont obse: $\mathrm{v}$ : à $77 \mathrm{~K}$ la variation d'intensité des raies d'émission $\mathrm{N}_{1}$ et $\mathrm{N}_{2}$ en fonction de l'excitation dans la régic $r$ de $4600-4900 \AA$. Ils ont mis en évidence des raies correspondant aux transitions $\left({ }^{4} \mathrm{~A}_{2}{ }^{4} \mathrm{~A}_{2}\right) \rightarrow\left({ }^{4} \mathrm{~A}_{2}{ }^{2} \mathrm{~T}_{2}\right)$.
D'autre part H. Engstrom et L. F. Mollenauer [12] utilisant la technique de chronospectroscopie et travaillant à la température de $77 \mathrm{~K}$ ont fait apparaître des bandes d'excitation de la raie $\mathrm{N}_{2}$ à 4650,4770 , 4850 et $6050 \AA$, qu'ils ont attribuées aux transitions $\left({ }^{4} \mathrm{~A}_{2}{ }^{4} \mathrm{~A}_{2}\right) \rightarrow\left({ }^{4} \mathrm{~A}_{2}{ }^{4} \mathrm{~T}_{1}\right)$ et $\left({ }^{4} \mathrm{~A}_{2}{ }^{4} \mathrm{~A}_{2}\right) \rightarrow\left({ }^{4} \mathrm{~A}_{2}{ }^{4} \mathrm{~T}_{2}\right)$ de cette paire. Ces travaux ont mis en évidence la contribution du pompage intrinsèque des niveaux excités des paires d'ions.

Dans ce présent travail nous donnons des résultats expérimentaux obtenus à $5,2 \mathrm{~K}$ et à $77 \mathrm{~K}$ et qui confirment le pompage intrinsèque des paires et mettent en évidence de nouveaux états d'énergie des paires d'ions.

L'étude de la transition ${ }^{4} \mathrm{~A}_{2} \rightarrow{ }^{4} \mathrm{~T}_{2}$ des ions $\mathrm{Cr}^{3}$. dans $\alpha-\mathrm{Al}_{2} \mathrm{O}_{3}$ peu dopé peut se faire à l'aide du spect. d'excitation de la raie $R_{1}$. Dans un rubis fortemcint dopé la variation de l'intensité des raies $\mathrm{N}_{1}$ ¿l $\mathrm{N}_{2}$. en fonction de l'excitation dans la même bande, nous fournit deux spectres différents de celu ${ }^{\prime}{ }^{\top}$ )tenu pour la raie $\mathbf{R}_{\mathbf{1}}$ d'un même échantillon. $L$ ' dispositif optique utilisé est constitué d'une source '‘ rayonnement (Lampe à Iode Mazda I.P.R. 250; $250 \mathrm{~W}, 24 \mathrm{~V}$ ) dont le filament est focalisé sur la fente d'entrée d'un monochromateur JOBIN-YVON 2M 23 de dispersion $12 \AA / \mathrm{mm}$, à la sortie de ce dernier la lumière est focalisée sur le cristal, sa longueur d'onde varie de $6100 \AA$ à $5750 \AA$. La lumière d'émission traverse un autre monochromateur identique au premier réglé sur l'une des raies $R_{1}, N_{\text {, ou }} \mathrm{N}_{2}$. Un photomultiplicateur type E.M.I. $944 \varepsilon$ 2.B. est placé à la sortie du deuxième monochromateur.

Nous avons effectué deux séries d'observations, 
la première à une température voisine de celle de l'hélium liquide $(5,2 \mathrm{~K})$, l'autre à la température de l'azote liquide. A chaque température on observe, pour deux échantillons dopés à 1,75 et $0,44 \%$ en poids de $\mathrm{Cr}_{2} \mathrm{O}_{3}$, les spectres d'excitation :

- $\mathrm{U}_{\mathrm{R}_{1}}$, correspondant à la transition ${ }^{4} \mathrm{~A}_{2} \rightarrow{ }^{4} \mathrm{~T}_{2}$ des ions $\mathrm{Cr}^{3+}$ isolés.

- $\mathrm{U}_{\mathrm{N}_{1}}$, correspondant à la transition

$$
\left({ }^{4} \mathrm{~A}_{2}{ }^{4} \mathrm{~A}_{2}\right) \rightarrow\left({ }^{4} \mathrm{~A}_{2}{ }^{4} \mathrm{~T}_{2}\right)
$$

des paires constituées par les $3^{\text {es }}$ plus proches voisins. - $\mathrm{U}_{\mathrm{N}_{2}}$, correspondant à la transition

$$
\left({ }^{4} \mathrm{~A}_{2}{ }^{4} \mathrm{~A}_{2}\right) \rightarrow\left({ }^{4} \mathrm{~A}_{2}{ }^{4} \mathrm{~T}_{2}\right)
$$

des paires constituées par les $4^{\text {èmes }}$ plus proches voisins.

A chaque spectre on superpose un spectre étalon fourni par une lampe spectrale au mercure, raie 5770 et $5790 \AA$.

Les figures 1 et 2 donnent les spectres obtenus à $5,2 \mathrm{~K}$ respectivement des cristaux dopés à 0,44 et $1,75 \%$ en poids de $\mathrm{Cr}_{2} \mathrm{O}_{3}$. La disposition adoptée sur ces figures permet la comparaison entre les spectres $U_{N_{1}}$ et $U_{N_{2}}$ des paires d'ions et le spectre $U_{R_{1}}$ des ions isolés. Dans la figure 1 qui donne les spectres d'excitation pour le cristal le moins concentré la raie à zéro-phonon des ions $\mathrm{Cr}^{3+}$ isolés apparaît nettement dans les spectres $U_{R_{1}}$ et $U_{N_{2}}$ à $5960 \AA$. On peut observer pour $\lambda>6000 \AA$, que le spectre $U_{N_{1}}$ présente deux raies de faible intensité situées à 6010 et $6084 \AA$ et que le spectre $U_{N_{2}}$ présente une raie située à $6055 \AA$. Ces raies n'existent pas dans le spectre $U_{\mathbf{R}_{1}}$ et elles sont particulièrement visibles pour le cristal le plus concentré à $5,2 \mathrm{~K}$ (Fig. 2).

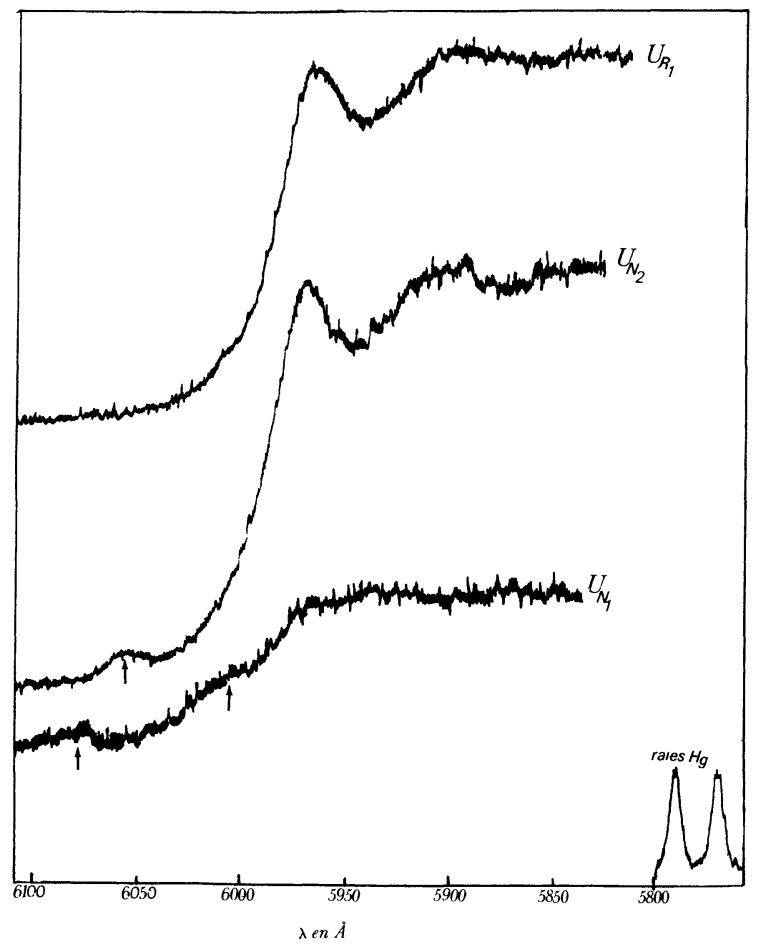

FIG. 1. - Spectres d'excitation des raies $R_{1}, N_{2}$ et $N_{1}$ du rubis dopé à $0,44 \%$ en poids de $\mathrm{Cr}_{2} \mathrm{O}_{3}$ à $5,2 \mathrm{~K}$.

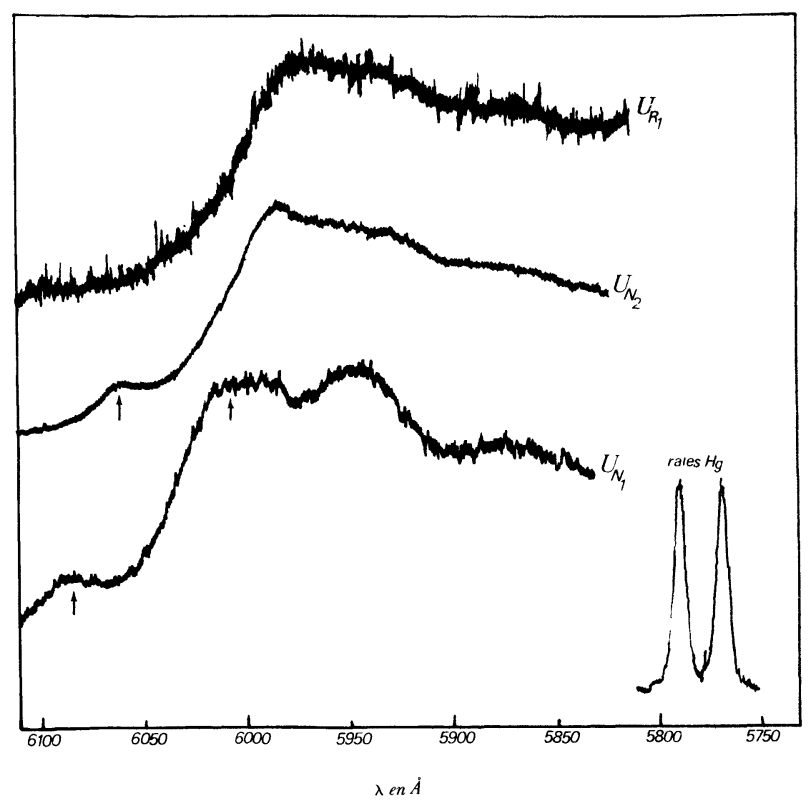

FIG. 2. - Spectres d'excitation des raies $R, N_{2}$ et $N_{1}$ du rubis dopé à $1,75 \%$ en poids de $\mathrm{Cr}_{2} \mathrm{O}_{3}$ à $5,2 \mathrm{~K}$.

Elles ne peuvent provenir alors que de transitions entre niveaux d'énergie de paire d'ions. En tenant compte de l'interaction électron-phonon dans l'état ${ }^{4} \mathrm{~T}_{2}$ des ions $\mathrm{Cr}^{3+}$ dans le rubis, on peut supposer que les raies des spectres $U_{N_{1}}$ et $U_{N_{2}}$ situées respectivement à 6084 et $6055 \AA$ sont des raies à zéro-phonon des paires d'ions correspondant aux transitions vers l'état où l'un des ions constituant la paire est dans le niveau ${ }^{4} \mathrm{~T}_{2}$, l'autre restant dans son état fondamental ${ }^{4} \mathrm{~A}_{2}$. La distance en énergie entre les raies à 6084 et $6010 \AA$ dans le spectre $U_{N_{1}}$ est de $202 \mathrm{~cm}^{-1}$ qui correspond bien à la distance entre la raie à zérophonon et la raie à un-phonon dans le spectre ${ }^{4} \mathrm{~A}_{2} \rightarrow{ }^{4} \mathrm{~T}_{2}$ des ions $\mathrm{Cr}^{3+}$ isolés. Il est donc probable que la raie à $6010 \AA$ corresponde à la raie à unphonon de la transition $\left({ }^{4} \mathrm{~A}_{2}{ }^{4} \mathrm{~A}_{2}\right) \rightarrow\left({ }^{4} \mathrm{~A}_{2}{ }^{4} \mathrm{~T}_{2}\right)$ des troisièmes voisins appariés. Les niveaux d'énergie $\left({ }^{4} \mathrm{~A}_{2}{ }^{4} \mathrm{~T}_{2}\right)$ se trouvent décalés vers les basses énergies par rapport aux niveaux ${ }^{4} \mathrm{~T}_{2}$ des ions $\mathrm{Cr}^{3+}$ isolés. On a calculé le décalage à l'aide des positions des raies à zéro-phonon, en utilisant la structure de l'état fondamental des paires [13]. En supposant que les transitions se font à partir du niveau d'énergie le plus bas on a trouvé que les niveaux $\left({ }^{4} \mathrm{~A}_{2}{ }^{4} \mathrm{~T}_{2}\right)$ des troisièmes voisins appariés et des quatrièmes voisins appariés se trouvent respectivement à $352 \mathrm{~cm}^{-1}$ et $265 \mathrm{~cm}^{-1}$ en dessous du niveau ${ }^{4} \mathrm{~T}_{2}$ des ions isolés.

On constate que l'intensité relative des raies à zéro-phonon des paires d'ions à $6084 \AA$ et $6055 \AA$ dans le spectre $U_{N_{1}}$ et le spectre $U_{N_{2}}$ respectivement, par rapport à celle de la raie à zéro-phonon des ions $\mathrm{Cr}^{3+}$ isolés, croît à $5,2 \mathrm{~K}$ et décroît à $77 \mathrm{~K}$ lorsque l'on augmente la concentration. Cette intensité relative mesure le rapport du pompage direct et du pompage par transfert à partir des ions $\mathbf{C r}^{3+}$ isolés. $\mathrm{R}$. C. Powell et al. [14] ont montré que la température de thermalisa- 
tion des niveaux excités des différents centres actifs dans le cristal décroît lorsque l'on augmente la concentration en ions $\mathrm{Cr}^{3+}$. Cela veut dire que la vitesse de transfert d'énergie entre les ions $\mathrm{Cr}^{3+}$ isolés et les paires diminue lorsque l'on abaisse la température du cristal et lorsque l'on diminue la concentration en ions $\mathrm{Cr}^{3+}$. On peut alors expliquer notre résultat expérimental. En effet la température de 5,2 $\mathrm{K}$ est inférieure à la température de thermalisation, et à cette température l'augmentation de la concentration a surtout pour effet d'augmenter le nombre de paires et par conséquent d'augmenter le signal correspondant au pompage direct. A $77 \mathrm{~K}$ dans le domaine de concentration considéré, l'augmentation de la concentration a principalement pour effet d'abaisser la température de thermalisation c'est-à-dire d'augmenter la vitesse de transfert ou le pompage par transfert.

Pour interpréter l'origine des niveaux $\left({ }^{4} \mathrm{~A}_{2}{ }^{4} \mathrm{~T}_{2}\right)$ des paires d'ions on propose deux hypothèses. La première consiste à considérer que le couplage des ions $\mathrm{Cr}^{3+}$ qui se fait par l'interaction d'échange lève la dégénérescence du niveau ${ }^{4} T_{2}$ et donne ainsi naissance au niveau $\left({ }^{4} \mathrm{~A}_{2}{ }^{4} \mathrm{~T}_{2}\right)$. Cette hypothèse a été introduite par Clogston [4] pour interpréter les niveaux $\left({ }^{4} A_{2}{ }^{4} A_{2}\right)$ des paires et généralisée par M. H. L. Pryce [8] et M. Naito [9] pour les niveaux $\left({ }^{4} \mathrm{~A}_{2}{ }^{2} \mathrm{E}\right)$. La seconde hypothèse que l'on propose est basée sur l'existence dans l'état ${ }^{4} \mathrm{~T}_{2}$ d'un effet Jahn-Teller avec des modes de vibration doublement dégénérés qui se traduit par trois puits de potentiel de même profondeur le long de trois directions de vibration [15-16]. Dans un rubis fortement dopé l'interaction élastique entre les deux ions d'une paire peut favoriser une direction de vibration et provoquer ainsi un abaissement du puits de potentiel correspondant à cette direction, l'état à zéro-phonon ${ }^{4} \mathrm{~T}_{2}$ des ions $\mathrm{Cr}^{3+}$ ne serait plus trois fois dégénéré comme pour les ions $\mathrm{Cr}^{3+}$ isolés, mais non dégénéré.

Nous effectuons actuellement des expériences qui permettent d'obtenir le spectre d'excitation correspondant au pompage direct isolé du spectre d'excitation correspondant au pompage par transfert. De plus grâce à ces nouvelles mesures nous espérons pouvoir choisir entre les deux hypothèses envisagées.

\section{Bibliographie}

[1] Deutschbein, O., Ann. Phys. 14 (1932) 712, $729 ; 20$ (1934) 828.

[2] Shawlow, A. L., Wood, D. L. and Clogston, A. M., Phys. Rev. Lett. 3 (1959) 271.

[3] Sugano, S. and Tanabe, Y., J. Phys. Soc. Japan 9 (1954) 735.

[4] Clogston, A. M., Rapport non publié.

[5] Mollenauer, L. F. and Schawlow, A. L., Phys. Rev. 168 (1968) 309.

[6] Kanskaya, L. M. and PrzhevuskiI, A. K., Sov. Phys.-Optics and Spectrosc. 26 (1969) 121.

[7] KaplyanskiI, A. A., Medvedev, V. N. and Przmevuskil, A. K., Sov. Phys.-J.E.T.P. Lett. 5 (1967) 347.

[8] Pryce, Non publié.

[9] NaIto, M., J. Phys. Soc. Japan 6 (1973) 1491.
[10] Imbusch, G. F., Phys. Rev. 153 (1967) 326.

[11] Kushida, T. and Tanaka, Y., Solid State Commun. 11 (1972) 1341.

[12] Engstrom, H. and Mollenauer, L. F., Phys. Rev. (B) 7 (1973) 1616.

[13] Powell, R. C. and Dibartolo, B., Phys. Stat. Sol. (a) 10 (1972) 315

[14] Powell, R. C., Dibartolo, B., Birang, B. and Naiman, C. S., Phys. Rev. 155 (1967) 296.

[15] Duval, E., Louat, R. and Lacroix, R., Phys. Stat. Sol. (b) 50 (1972) 627

[16] Louat, R., Lacroix, R., Duval, E. and Champagnon, B., Phys. Stat. Sol. (b) to be published. 